\title{
A Memory Based Model for Knowledge Organization and Sharing in Knowledge Grid
}

\author{
Jing Li \\ College of Economics \& Business Administration, Beijing Normal University, Beijing \\ 100875, P.R. China lijing0622@buaa.edu.cn
}

\begin{abstract}
The knowledge grid is an intelligent interconnection environment that enables people to effectively manage knowledge resources. To enhance the acquirement and sharing of critical knowledge in knowledge grid, this paper proposes a new knowledge model -- memory grid. Memory grid incorporates memory theory of psychology into knowledge grid to deliver plenty of context information. Based on the intelligent interconnection and increasing mechanism of memory, memory grid not only supports the organization of explicit knowledge, but also enables the acquirement of knowledge through memory transformation mode. Memory can be described by some entities along three dimensions (business, agent and knowledge), so memories can be semantically related with each other. Based on the above model, a web-based knowledge management system is developed for a Chinese enterprise to facilitate its knowledge management in the collaborative commerce environment.
\end{abstract}

Keywords: Knowledge management, Knowledge sharing, Knowledge grid, Memory, Knowledge management system

\section{INTRODUCTION}

Knowledge management has already become the most important instrument to build the enterprise competence. It aims at transforming the knowledge into creativity through knowledge acquisition, sharing, application and creation [1]. The Knowledge Grid is an intelligent interconnection environment that enables people or virtual roles to effectively manage explicit knowledge resources. It also provides on-demand services to support innovation, cooperative teamwork, problem solving, and decision making $[2,3]$.

Knowledge modeling is a fundamental step for knowledge management system (KMS) development in knowledge grid environment. Knowledge repository is one of the main storage technologies and the core of KMS [4]. Most knowledge repositories are built around explicit knowledge objects classified by subject area, but it is impossible to dig up tacit knowledge in it.

Memory is considered by some psychologists as one of the most basic cognitive processes. There are two different kinds of memory stores: short-term memory and long-term memory. Tulving argued that long-term memory is made of two different 
yet interacting systems: semantic memory for general knowledge and episodic memory for events $[5,6]$.

This paper presents a new knowledge model, memory grid, which incorporates memory theory of psychology into knowledge grid technology to deliver plenty of context information. Memory grid not only supports the organization of explicit knowledge, but also enables the acquirement of some tacit knowledge through memory transformation mode.

\section{THE MEMORY GRID}

In this section, we introduce the concept and basic mechanism of memory grid based on the knowledge grid theory.

Definition 1 Memory is retaining of the history about knowledge operations which are brought to bear on business or problem in the organization.

Definition 2 Memory grid is an integrated memory environment that enables people or roles to effective capture, publish, share and manage memory resources. It delivers plenty of context information, intelligent interconnection and increasing mechanism for memory resources to support knowledge management and decision making in the cooperative teamwork.

Compared with the existing research on knowledge grid, memory grid has the following characteristics. Firstly, memory grid provides a harmonious container, i.e. memory, to acquire and manage knowledge distributed around the world. Memory records people's decisions and operations about knowledge, which involves plenty of context information. Secondly, memories can associate with each other because of the semantic relationships between them, which makes relevant memories distributed around the world can intelligently cluster together and fused to provide on-demand knowledge services with underlying reasoning and explanations. Thirdly, memory can also increase naturally, which enables association among memories more wealthy. Fourthly, Memory is domain oriented. In a specific domain, people or virtual roles can share memory and enjoy reasoning services in a single semantic space. Finally, memory grid takes more considerations on management and humanism because business and social networks are embedded into memory grid.

It is the memory that makes memory grid different from general knowledge grid. Memory transformation is the foundational mode in memory grid. Based on memory transformation, memory grid can support to acquire some tacit knowledge, which is just hidden behind the usage pattern of explicit knowledge. Figure 1 shows the memory transformation mode from short-term memory to long-term memory, from organizational episodic memory (OEM) to organizational semantic memory (OSM).

\section{SEMANTICS IN MEMORY GRID}

Memory is context-rich essentially. In order to explicate memory and express it in a standard structure, we describe the memory from three dimensions: agent, business and knowledge. 


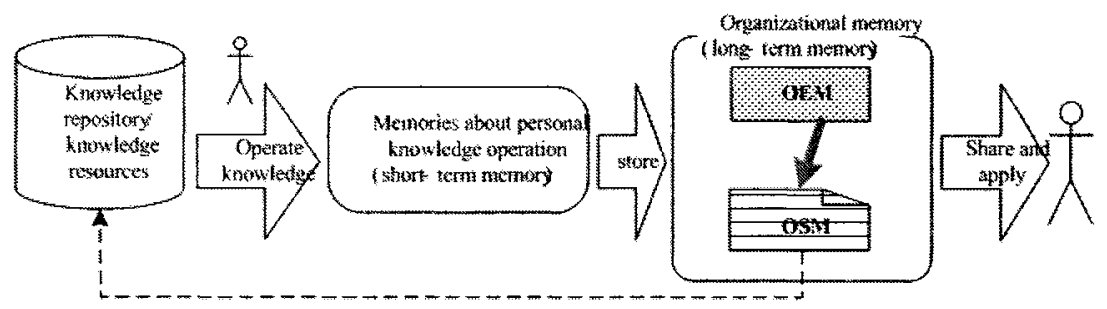

Figure 1. Memory Transformation Mode in Memory Grid

Definition 3 A memory fragment is the fundamental underlying construct of the memory grid and serves as the basic unit of analysis for all operations defined on memory grid. It is defined as a 3-tuple, $\langle D, E, f\rangle$, where the three components indicate the characteristics of the fragment. These characteristics are:

- $\mathrm{D}$ is a set of 3 dimensions $\left\{d_{1}, d_{2}, d_{3}\right\}$, where $d_{1}$ is the dimension of business, $d_{2}$ is the dimension of agent and $d_{3}$ is the dimension of knowledge.

- $\mathrm{E}$ is a set of $n$ entities $\left\{e_{1}, e_{2}, \cdots e_{i}, \cdots, e_{n}\right\}$ where each $e_{i}$ is an entity name.

- $f$ is a one-to-one mapping, $f: D \rightarrow 2^{E}$ which maps a set of entities to each dimension.

In order to analyze the memories, we define some basic semantic relationships and operations which act on the fragment structure.

Definition 4 Relationship is a particular type of semantic connection existing between related entities in the memories.

We define some types of relationships between entities in Table 1.

Table 1. Relationships between Entities

\begin{tabular}{|l|l|}
\hline \multicolumn{1}{|c|}{ Type } & \multicolumn{1}{|c|}{ Relationship Name } \\
\hline Simple relationship & Equal-to, Similar-with, Null \\
\hline Basic relationship & $\begin{array}{l}\text { Associate-with, Compose, Generalize, Depend-on, } \\
\text { Instance-of }\end{array}$ \\
\hline $\begin{array}{l}\text { Extended } \\
\text { relationship }\end{array}$ & $\begin{array}{l}\text { Associate-with: For instance, employing, applying, owing } \\
\text { Depend-on: Sequential dependence, Data dependence, } \\
\text { Function Dependence }\end{array}$ \\
\hline
\end{tabular}

Base on the above relationships between entities, memories can be linked with each other. Thus memory association can be realized in memory grid. In psychology, association is a mental connection or relation between thoughts, feelings, ideas, or sensations. Here we give a basic mechanism of association in memory grid.

Definition 5 Association in memory grid is a process to search for connection paths linked to a given memory or an entity in a memory.

So association in memory grid is a process of graph traverse. We can adopt breadth-first-search algorithm and depth-first-search algorithm to implement the association process in memory grid. 
Association provides a basic mechanism for memory analysis and knowledge modeling in memory grid. Before we have researched on some knowledge modeling methods based on memory grid. In [7] we take the people's tagging as a kind of memory and construct knowledge map through memory analysis.

\section{THE ARCHITECTURE OF MEMORY GRID BASED KMS}

Memory grid provides a new thought for the development of KMS and especially for knowledge modeling in knowledge grid. Here we give a three-layered architecture of memory grid based KMS, as is shown in Figure 2, which is developed for a Chinese enterprise.

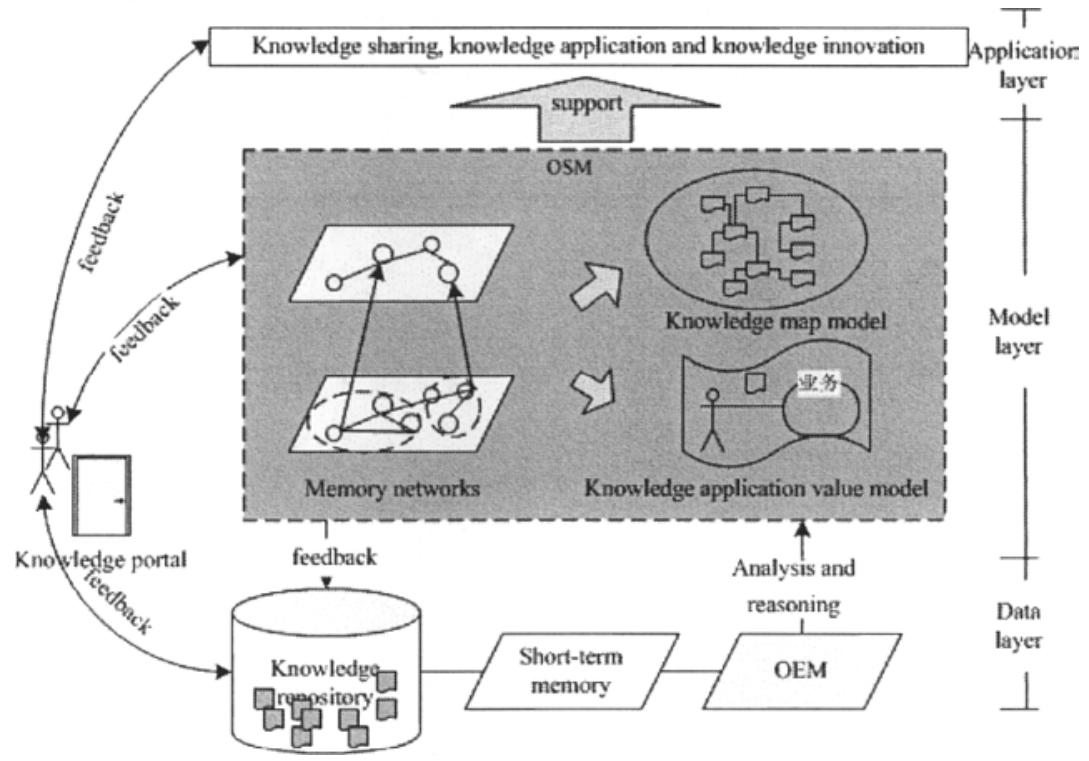

Figure 2. The Architecture of Memory Grid Based KMS

1. Data layer. The major task in this layer is to store information about knowledge usage into memory grid. Traditional knowledge repository is integrated into memory grid. Knowledge repository stores large amount of knowledge items, while memory grid records memories, which occurs when people in some business context operate on the knowledge items stored in the knowledge repository.

2. Model layer. Through the semantic analysis defined in Section 3, we construct some knowledge models. So far, we have developed the knowledge map model and knowledge value model. With the implementation of the system, we will obtain more memories from users and acquire more knowledge models from these memories. 
3. Application layer. Application layer includes all kinds of knowledge services that help to realize knowledge creation, acquirement, sharing and usage, such as document inquiry, case retrieval and training. As the system records memories from multiple distributed agents, it is necessary to develop a knowledge portal for all agents to access the knowledge items and record their operations.

\section{CONCLUSIONS}

This paper presents memory grid model. Memory grid is an integrated memory envitonment. Memories are context-rich, intelligent interconnected and increasing. It helps to support the management of explicit knowledge and the acquirement of some tacit knowledge. This model has been implemented in the development of a KMS for a Chinese enterprise.

\section{REFERENCES}

1. M. Yogesh, Knowledge Management and New Organization Forms: a Framework for Business Model Innovation, Information Resources Management Journal. Volume 13, Number 1, pp.5-14 (2000).

2. H. Zhuge and J. Liu, A Fuzzy Collaborative Assessment Approach for Knowledge Grid, Future Generation Computer Systems. Volume 20, Number 1, pp.101-111 (2004).

3. H. Zhuge and X. Luo, Automatic Generation of Document Semantics for the E-science Knowledge Grid, The Journal of Systems and Software. Volume 79, Number 7, pp.969. 983 (2006).

4. T.H. Davenport, D.W. DeLong, and M.C. Beers, Successful Knowledge Management Projects, Sloan Management Review. Volume 39, Number 2, pp.43-58, (1998).

5. E. Tulving, Episodic and semantic memory, in Organization of Memory, eds. E. Tulving and E. Donaldson (Academic Press: New York, NY, 1972), pp.381-403.

6. E. Tulving, Ecphoric processes in recall and recognition, in Recognition and Recall, eds. J. Brown (Wiley: London, 1976), pp.37-73.

7. L. Liu, J. Li, and C.G. Lv, A Method for Enterprise Knowledge Map Construction Based on Social Classification, in Proc. of The IFIP TC8 International Conference on Research and Practical Issues of Enterprise Information Systems, eds. A.M. Tjoa, L. Xu, and S.S. Chaudhry (Springer: New York, 2006), pp.541-551. 